\title{
Monitoring inflammation and airway remodeling by fluorescence molecular tomography in a chronic asthma model
}

\author{
Fabio Stellari ${ }^{1}$, Angelo Sala ${ }^{2,3}{ }^{*}$, Francesca Ruscitti ${ }^{4}$, Chiara Carnini ${ }^{1}$, Prisco Mirandola ${ }^{4}$, Marco Vitale ${ }^{4}$, \\ Maurizio Civelli ${ }^{1}$ and Gino Villetti ${ }^{1}$
}

\begin{abstract}
Background: Asthma is a multifactorial disease for which a variety of mouse models have been developed. A major drawback of these models is represented by the transient nature of the airway pathology peaking $24-72 \mathrm{~h}$ after challenge and resolving in 1-2 weeks. We characterized the temporal evolution of pulmonary inflammation and tissue remodeling in a recently described mouse model of chronic asthma (8 week treatment with 3 allergens: Dust mite, Ragweed, and Aspergillus; DRA).
\end{abstract}

Methods: We studied the DRA model taking advantage of fluorescence molecular tomography (FMT) imaging using near-infrared probes to non-invasively evaluate lung inflammation and airway remodeling. At 4, 6, 8 or 11 weeks, cathepsin- and metalloproteinase-dependent fluorescence was evaluated in vivo. A subgroup of animals, after 4 weeks of DRA, was treated with Budesonide (100 $\mu \mathrm{g} / \mathrm{kg}$ intranasally) daily for 4 weeks.

Results: Cathepsin-dependent fluorescence in DRA-sensitized mice resulted significantly increased at 6 and 8 weeks, and was markedly inhibited by budesonide. This fluorescent signal well correlated with ex vivo analysis such as bronchoalveolar lavage eosinophils and pulmonary inflammatory cell infiltration. Metalloproteinase-dependent fluorescence was significantly increased at 8 and 11 weeks, nicely correlated with collagen deposition, as evaluated histologically by Masson's Trichrome staining, and airway epithelium hypertrophy, and was only partly inhibited by budesonide.

Conclusions: FMT proved suitable for longitudinal studies to evaluate asthma progression, showing that cathepsin activity could be used to monitor inflammatory cell infiltration while metalloproteinase activity parallels airway remodeling, allowing the determination of steroid treatment efficacy in a chronic asthma model in mice.

Keywords: Animal chronic asthma model, Fluorescence molecular tomography, Inflammatory cell infiltration, Airways remodeling

\section{Background}

Asthma is a multifactorial and multicellular disease, and relevant animal models are needed for a better understanding of the pathophysiology of asthma and for the development of new therapeutic approaches. A variety of mouse models have been described that capture specific

\footnotetext{
*Correspondence: angelo.sala@unimi.it

2 Dipartimento di Scienze Farmacologiche e Biomolecolari, Università degli Studi di Milano, Via Balzaretti 9, 20133 Milan, Italy

Full list of author information is available at the end of the article
}

inflammatory mechanisms of asthma [1], but major drawbacks are represented by the transient nature of the airway pathological response, that peaks at $24-72 \mathrm{~h}$ after sensitization-challenge and resolve in 1-2 weeks [2, 3], and the development of tolerance after chronic exposure to single allergens $[4,5]$.

Recently a mouse model of chronic asthma has been described by Goplen [6], where the sensitization of Balb-C mice for 8 weeks with a combination of 3 natural allergens relevant for humans (dust mite, ragweed, and aspergillus: DRA) breaks through tolerance and 
establishes chronic features of asthma. Chronic DRA administration leads to inflammation and epithelial hyperplasia reaching a maximum score at the end of the sensitization protocol (week 8), with pathological changes that persist for 2-3 weeks after the last allergen exposure, even if with a lower degree of severity. Unfortunately, the conventional assessments of asthma in mice often rely on invasive measures of pulmonary function, ex vivo characterization of pulmonary cellular infiltration and observation of anatomical changes, such as airway remodeling due to inflammation, an approach that, while used extensively and highly validated, preclude the possibility of repeated, longitudinal assessment of test animals. Recently, non invasive MRI and micro-CT techniques have been applied to longitudinal monitoring of airway remodeling in murine asthma models $[7,8]$, and to assess lung fluid accumulation as well as the anti-inflammatory effects of $\mathrm{S} 1 \mathrm{P}(1)$ receptor activation in allergen-induced lung inflammation $[9,10]$, opening the possibility to noninvasively visualize and quantitate inflammatory features of mouse asthma models in vivo.

Clinical visualization of asthma has largely been based on imaging methods such as high resolution CT, positron emission tomography (PET), single photon emission computed tomography (SPECT), and magnetic resonance imaging (MRI), that have been applied to the visualization of mechanical and physiological responses, for example diaphragmatic motion, pulmonary perfusion and ventilation as well as airway remodeling [11-14]. The techniques used in clinical imaging such as MRI, CT and PET, have been further adapted to small animal research and used in murine models of asthma [7-9, 15, 16], to serve as a preclinical, translational step between basic discovery and clinical practice, while imaging techniques developed in experimental settings may also slowly make their way into the clinical practice, especially in the context of intraoperative activities [17, 18]. Among these latter, fluorescence molecular tomography (FMT) is a relatively new tomographic imaging technique involving principles similar to X-ray CT but based on the use of target-specific molecular fluorescent reporters and volumetric reconstruction of light emitted from the probes [19].

In the present paper we report on the possibility to use near-infrared imaging agents, in combination with FMT imaging, for the non-invasive quantitative imaging of mouse lung inflammation in the DRA mouse model. The results obtained with both a cathepsin-activable and a metalloproteinase-activable fluorescent agents well correlated with ex vivo read-outs such as bronchoalveolar lavage cell analysis, pulmonary inflammatory cell infiltrations, and histological analysis of airway epithelium hyperplasia and collagen deposition, allowing differential monitoring of cellular infiltration or tissue remodeling during the inflammatory response associated with DRA.

\section{Methods \\ Animals}

Female Balb-C (7-8 week-old) mice were purchased from Charles River Laboratories Italy (Calco, Lecco, Italy). Animals were maintained under conventional housing conditions. Prior to use animals were acclimated for at least 5 days to the local vivarium conditions (room temperature: $20-24{ }^{\circ} \mathrm{C}$; relative humidity: $40-70 \%$ ), having free access to standard mouse chow and tap water. All animal experiments described herein were approved by the intramural animal-welfare committee for animal experimentation of Chiesi Farmaceutici and comply with the European Directive 2010/63 UE, Italian D.Lgs $26 / 2014$ and the revised "Guide for the Care and Use of Laboratory Animals" [20].

\section{DRA induction protocol}

Mice, under light isoflurane anesthesia, were treated intranasally (i.n.) with a $30 \mu \mathrm{L}$ volume $(15 \mu \mathrm{L}$ into each nostril) of DRA solution containing $5 \mu \mathrm{g}$ of dust mite (extracts of Dermatophagoides farinae, Greer Laboratories, NC, USA), $50 \mu \mathrm{g}$ of ragweed (extracts of Ambrosia artemisiifolia, Greer Lab) and $5 \mu \mathrm{g}$ of Aspergillus (extracts of Aspergillus fumigatus, Greer Lab), twice a week for 8 weeks. The control group received $30 \mu \mathrm{L}$ (15 $\mu \mathrm{L}$ into each nostril) of saline i.n. using the same protocol. Among the animals undergoing DRA challenge a group was treated with $100 \mu \mathrm{g} / \mathrm{Kg}$ budesonide i.n. 6 day/ week for 4 weeks starting at the end of week 4 . Eight mice were used for every group and the experiment was replicated three times.

\section{In vivo fluorescent imaging}

DRA-challenged and control mice were injected intravenously with ProSense680 (Perkin Elmer, Hanover, CA, USA) or MMPSense680 (Perkin Elmer) at 80 nmoles/ Kg on weeks 4, 6, 8 (i.e., few minutes after the last intranasal administration of DRA), and 11, and imaged $24 \mathrm{~h}$ after probe injection. ProSense680 is a fluorescent in vivo imaging agent that is activated by proteases such as Cathepsin B, L, S and Plasmin, and MMPSense680 is a fluorescent in vivo imaging agent that is activated by matrix metalloproteinases (MMPs) including MMP-2, -3, -9 and -13. ProSense 680 and MMPSense 680 are optically silent in its unactivated state and becomes highly fluorescent following protease-mediated cleavage.

At the 4 weeks time point, animals were randomised to budesonide and control treatments according to the intensity of the fluorescent signal resulting from ProSense680. 
Mice were anaesthetized using isoflurane and depilated to minimize fur interference with fluorescent signal. Depilatory cream was applied thickly on skin over the upper torso (front, back, and sides) of each mouse, rinsed off with warm water, and reapplied until all fur had been removed.

DRA-challenged and control mice were then imaged using the FMT 2500 in vivo imaging system (VisEn Medical, Inc., Bedford, MA, USA). Briefly, the anesthetized mice were carefully positioned in the imaging cassette, which was then placed into the FMT imaging chamber. A near infrared (NIR) laser diode transilluminated (i.e., passed light through the body of the animal to be collected on the opposite side) the thorax region, with signal detection occurring via a thermoelectrically cooled charge-coupled device camera placed on the opposite side of the imaged animal. Appropriate optical filters allowed collection of both fluorescence and excitation data sets, and the multiple source-detector fluorescence projections were normalized to the paired collection of laser excitation data. The entire image acquisition sequence took approximately 4-5 min per mouse.

The collected fluorescence data were reconstructed by FMT 2500 system software version 2.2 (PerkinElmer) for the quantification of three-dimensional fluorescence signal within the lungs. Three-dimensional regions of interest were drawn in the upper torso encompassing the lung region (below the manubrium and above the liver) excluding $2 \mathrm{~mm}$ ventrally and $3 \mathrm{~mm}$ dorsally. The total amount of lung fluorescence (in picomoles) and the fluorescence volume (in cubic millimeters) were automatically calculated from the fluorescence of internal standards generated with known concentrations of appropriate NIR dyes. The same mice were submitted to both probes, and have been evaluated at 4-6-8 and 11 weeks: first the animals were injected with ProSense, and after 24-36 h MPPSense 680 was injected, after screening the residual fluorescence signal by FMT.

\section{Bronchoalveolar lavage fluid collection, cell counting and cytokine determination}

Animals were anaesthetised with isoflurane and sacrificed by bleeding from the abdominal aorta. Eight mice were used for every time points and the experiment was replicated three times.

Bronchoalveolar lavage was performed and bronchoalveolar lavage fluids (BALFs) were collected as previously described [21]. Briefly, mouse tracheas were cannulated with a 18-gauge angiocathether. BALF was performed by gently washing the lungs with $0.6 \mathrm{~mL}$ sterile solution [Hank's balanced salt solution (HBSS) $\times 10$; (EDTA) $100 \mathrm{mM}$; 4-(2-hydroxy-ethyl)-1-piperazineethansulphonic acid (HEPES) $1 \mathrm{mM}$; distilled water] for three times in the bronchial tree and collected for subsequent analysis. After centrifugation at $400 \mathrm{~g}$ for $10 \mathrm{~min}$, the BAL supernatants were frozen at $-80{ }^{\circ} \mathrm{C}$. The cell pellet was re-suspended in $0.2 \mathrm{~mL}$ of PBS. Cell number and differential count were performed with an automatic cell counter (Dasit XT 1800J, Cornaredo, Milano, Italy). Supernatants were used for simultaneous quantitation of multiple cytokines/chemokines using a Bio-Plex ${ }^{\mathrm{TM}}$ Cytokine Assay Kit (Bio-Rad Laboratories, Segrate, Milano, Italy) according to the manufacturer instructions.

\section{Flow cytometric analysis of BAL cells}

Twenty-four hours after the last DRA challenge, BAL was performed and cells were subsequently analyzed for surface markers. Cells were labelled in phosphate buffered saline (PBS, Euroclone) and $0.5 \%$ bovine serum albumine (BSA, Milteny Biotech) with fluorochrome-labeled monoclonal antibodies: anti mouse CD 45 PE-Cy5 (BD Pharmigen), anti mouse F4/80 Alexa 488 (AbD Serotec), anti mouse Lys6G (BD Pharmigen), anti mouse CD11b PE-Cy7 (BD Pharmigen) and appropriate isotype controls for $30 \mathrm{~min}$ at RT in the dark. Cells were washed before and after the staining and resuspended in $300 \mu \mathrm{L}$ of PBS/BSA. Samples were collected on a FACS Canto II (2 lasers, 6 colors, Becton-Dickinson) and analyzed using Diva 7 software. Mean Fluorescence Intensity (MFI) was determed on a statistically significant number of cells each sample. To positively select all leukocytes in BAL and discard debris, gating was performed on CD45 positive cells. Anti-mouse F4/80 was used to discriminate granulocyte population, including eosinophils and neutrophils, from macrophages. Lymphocytes were gated out based on forward scatter (FSC) and side scatter (SSC). Moreover anti-mouse $\mathrm{GR}^{+}{ }^{+}$was used to negatively gate out all neutrophils. FACS analysis finally quantitated CD11b surface activation marker expression on the remaining population of monocytes/macrophages characterized as $\mathrm{CD}_{4} 5^{+} \mathrm{F} 4 / 80^{+} \mathrm{GR} 1^{-}$cells.

Histological processing of lung and morphometric analysis Eight mice were used for every time points and the experiment was replicated three times. Lungs were collected, gently inflated with $10 \%$ neutral buffered formalin (about $1 \mathrm{~mL}$ ) through a tracheal blunt needle, immersed in formalin and embedded longitudinally en bloc. Histological sections $(3 \mu \mathrm{m})$ were stained with hematoxylin/ eosin, alcian blue/PAS and Masson's trichrome stains. In the haematoxylin/eosin stained sections, the following parameters were evaluated: airways epithelium hypertrophy/hyperplasia, perivascular/peribronchial/interstitial inflammation, pulmonary tissue infiltration of inflammatory cells, and vascular smooth muscle hypertrophy. Sections were semi-quantitatively scored as follows: 0, absent; 1 , minimal; 2 , slight; 3 , moderate. 
The alcian blue/PAS histochemical stain was used in order to characterize the glycoprotein component of goblet cells in the respiratory epithelium. Sections were semi-quantitatively scored, based on the amount of alcian blue/PAS positive cells observed, as follows: 0: absent/rare; 1: minimal (1-30\%); 2: slight (31-60\%); 3: moderate $(>60 \%)$.

The Masson's trichrome histochemical stain was used to evaluate the amount of collagen deposition around the airways. Sections were semi-quantitatively scored as follows: 0 , absent; 1 : minimal (1-30\% of structures involved); 2: slight (31-60\% of structures involved); 3 : moderate ( $>60 \%$ of structures involved).

To evaluate the pulmonary inflammatory infiltrate and the airway epithelial hyperplasia in lung specimens, seven airways per mouse were taken into consideration. In order to be considered suitable for this evaluation, airways needed to be completely intact and entirely contained in a $200 \times$ microscopic field. Oblong airways were excluded from the analysis.

Pulmonary inflammatory infiltrate and airway epithelial hyperplasia, quantified in digitally acquired images analyzed with SIS software (Soft Imaging System $\mathrm{GmbH}$, Münster, Germany), were measured as the area of inflammatory infiltrates and the area of epithelium (in square micrometers) divided by the airway's basement membrane perimeter (in micrometers).

In detail, airway measurements included the perimeter of the basal membrane of the epithelium (AP), the area of the region circumscribed by the basal membrane (AA), the area of the lumen (AL) and the area covered by inflammatory infiltrates (AII). The airway epithelial hyperplasia score, corresponding to (AA - AL)/AP and the pulmonary inflammatory infiltrate score, corresponding to AII/AP, were calculated for each airway. A mouse mean score and a mean total score for each group of mice were then calculated.

\section{Data analysis}

Data were expressed as the mean and s.e. of the mean of $\mathrm{n}$ animals. The inhibition values reported in different Figures represents the comparison, at a given time point, between the increase of the observed values above the dashed line (representing the average value of control animals receiving saline instead of DRA) reported in the absence and that reported in the presence of budesonide. Statistical analysis was performed using one-way ANOVA followed by Dunnett's t test for multiple comparison (PRISM Statistical software v 4.0.3). $P<0.05$ was considered a level of statistical significance.

\section{Results}

In agreement with the results of Goplen et al. [6], treatment with DRA for 8 weeks, twice a week, established an inflammatory pulmonary phenotype characterized by a massive inflammatory cells infiltration in the airways, as shown by the marked increase in the number of total white cells in BALFs (Fig. 1a), with eosinophils representing the prevalent population (Fig. 1b), together with lymphocytes (Fig. 1c) and neutrophils (Fig. 1d). Treatment with budesonide i.n. daily, starting at 4 weeks and continuing up to 8 weeks of DRA, markedly inhibited eosinophils and lymphocytes recruitment at 6 weeks (85 and $64 \%$ inhibition when compared to saline-treated animals, respectively) and 8 weeks (72 and $52 \%$ inhibition when compared to saline-treated animals, respectively) (Fig. 1b, c). The inhibition of neutrophil infiltration resulted much less pronounced and was statistically significant only at the 6 weeks time-point (Fig. 1d). Inflammatory cell infiltrate substantially resolved at 11 weeks, where the total number of cells was not statistically different from saline-treated controls.

Infiltration of monocytes into the airways following DRA chronic exposure resulted in increased number of monocyte/macrophages in BALFs (Fig. 2a), with an increased expression of the integrin CD11b (Fig. 2b, c); treatment with budesonide significantly inhibited the infiltration of monocytes, reducing the number of monocytes/macrophages as well as their expression of the integrin adhesion molecule CD11b (Fig. 2a, b, d).

Out of 23 cytokines evaluated in BALFs, 6 resulted elevated upon DRA administration, and budesonide statistically inhibited the increase of IL-17 both after 6 and 8 weeks of DRA. Interestingly, the inhibition of TNF $\alpha$ and IL-8 resulted statistically significant only after 6 weeks of DRA, while IL-5 and IL-10 concentrations were markedly affected only after 8 weeks of DRA (Fig. 3).

In vivo imaging of the cathepsin-activated near infrared fluorescent probe ProSense680, carried out at different times on separate groups of animals, in parallel with the groups used for BALFs fluid collection and histological analysis, showed a statistically significant increase in fluorescent signal as a result of DRA administration (Fig. 4a, b), an increase that also resulted inhibited by budesonide treatment by 85 and $60 \%$ at 6 and 8 weeks, respectively (Fig. $4 \mathrm{a}, \mathrm{b}$ ). The fluorescent signal well correlated with the number of inflammatory cells in BALFs observed at 8 weeks $\left(r^{2} 0.997\right.$; Fig. $\left.4 c\right)$, while at 11 weeks it was reduced near to basal values (Fig. 4b), in parallel with the observed decrease of inflammatory cell infiltration into the airways (Fig. 1a). Ex vivo imaging of excised lungs confirmed that the fluorescence observed upon 

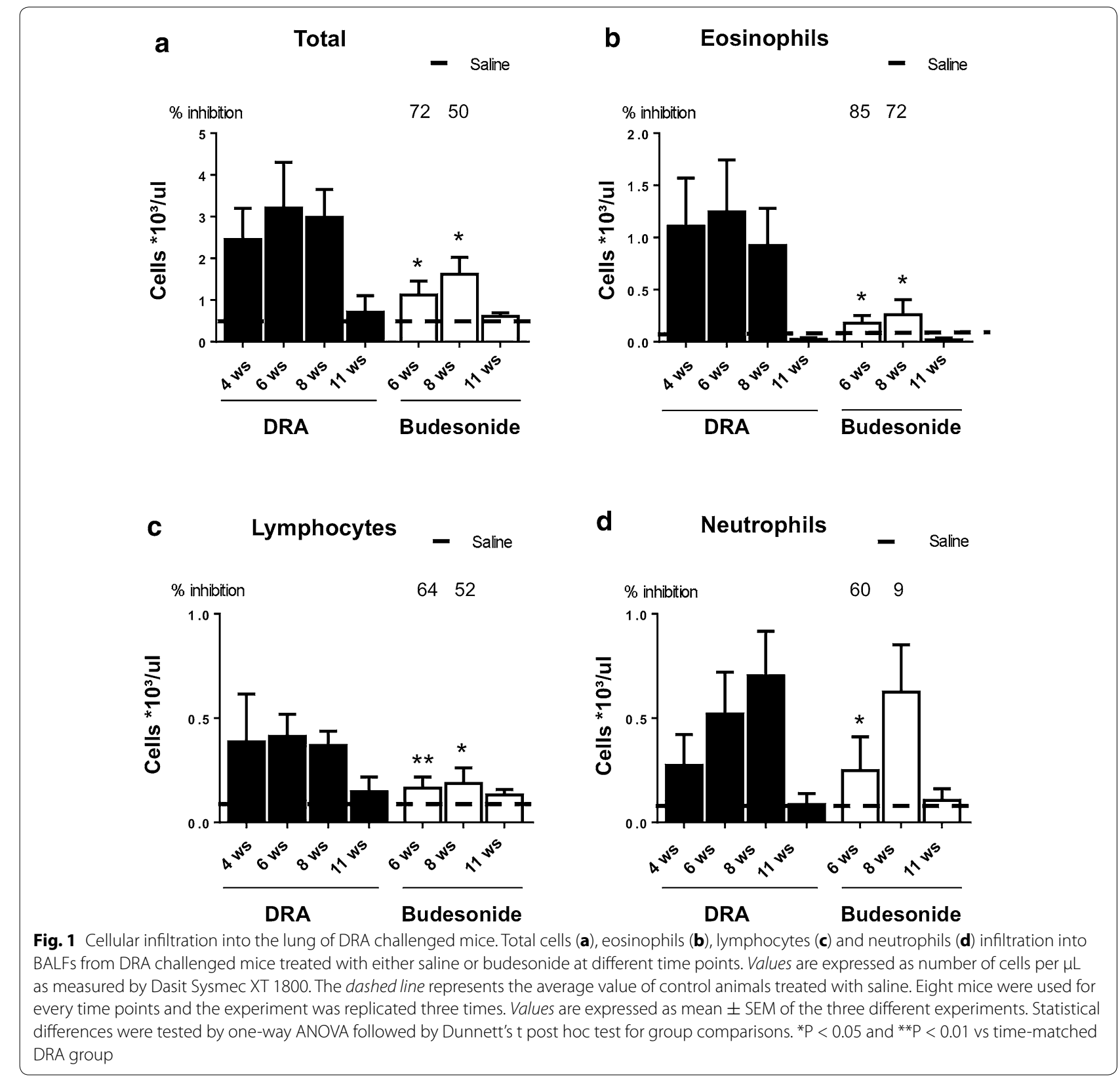

ProSense and MMPSense administration was associated with the lungs (Additional file 1: Figure S1).

Changes in the results of the histological analysis of inflammatory cell infiltrate in saline, DRA and budesonide treated animals (Fig. 5a), at different time points, well correlated with the number of inflammatory cells in BALFs as well as with the ProSense680 fluorescence signals.

The quantitative imaging of fluorescence resulting from the metalloproteinase-activated NIRF probe MMPSense680 also showed a significant increase in DRA animals at 8 weeks, but, unlike ProSense680, it did not resolve at 11 weeks (Fig. 6a, c), when inflammatory cells were not present anymore (Fig. 1a). Although the pmoles of fluorescence signal calculated in the ROI region may produce pictures suggesting a distribution not perfectly symmetrical, this is mostly due to light scattering and we could not discriminate if a single lobe was carrying more inflammation. On the other end, histological analysis of collagen deposition at 11 weeks showed score values still significantly increased in DRA animals when compared to saline-treated animals, in agreement with the ongoing 
a

Monocytes

$\%$ inhibition

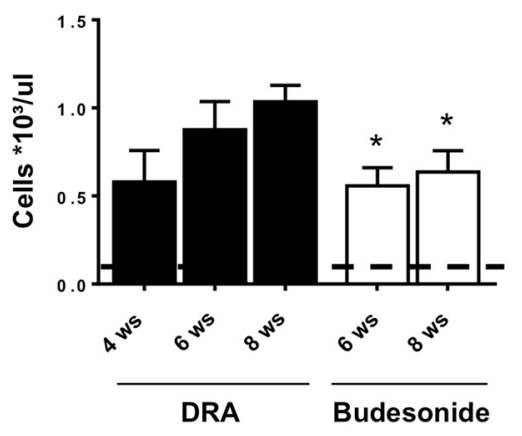

C

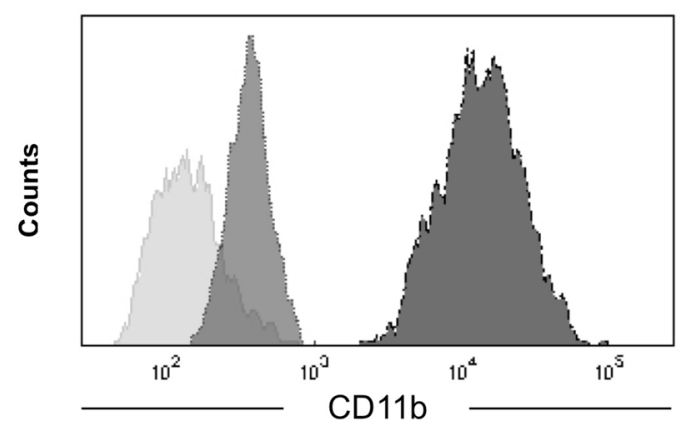

ISOTYPE CONTROL

- SALINE

- DRA b CD 11 b expression

$\begin{array}{lll} & - & \text { Salin } \\ \% \text { inhibition } & 61 & 71\end{array}$

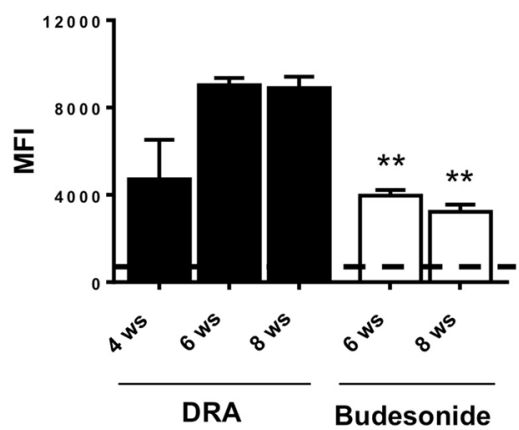

d DRA + Budesonide

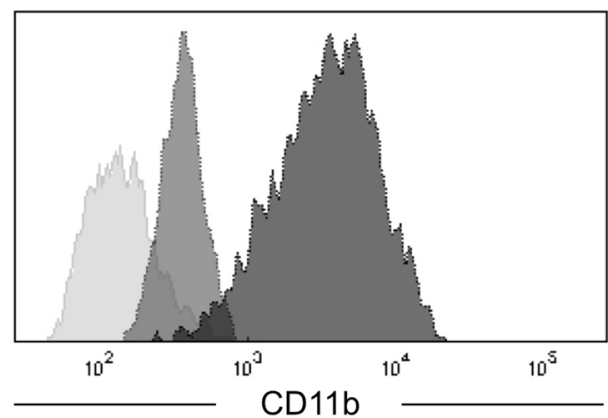

ISOTYPE CONTROL

- SALINE

- DRA+BUDESONIDE

Fig. 2 a Number of monocytes/macrophages in BALF from control and DRA challenged mice treated with either saline or budesonide at different time points. Values are expressed as number of cells per $\mu \mathrm{L}$ as measured by Dasit Sysmec XT 1800. The dashed line represents the average value of control animals treated with saline. $\mathbf{b}$ Expression of CD1 1 b integrin surface marker on monocytes/macrophages present in BALFs as measured by FACS analysis in BALF from DRA challenged mice treated with either saline or budesonide at different time points. Values are expressed as Mean Fluorescence Intensity (MFI). The dashed line represents the average value of control animals treated with saline. c Representative picture of CD1 1b integrin surface marker on monocytes measured by FACS analysis; CD1 1 b expression of mice treated with either saline (dark grey) or 8 weeks of DRA (black), with isotype control being depicted in light grey. $\mathbf{d}$ Representative picture of CD11 b integrin surface marker on monocytes measured by FACS analysis; CD 11 b expression of mice treated with either saline (dark grey) or 8 weeks of DRA and budesonide beginning after 4 weeks of DRA (black), with isotype control being depicted in light grey. Eight mice were used for every time points and the experiment was replicated three times. Values are expressed as mean \pm SEM of the three different experiments. Statistical differences were tested by one-way ANOVA followed by Dunnett's t post hoc test for group comparisons. ${ }^{* P}<0.05$ and ${ }^{* * P}<0.01$ vs time-matched DRA group

process of airways remodeling that is known to outlast inflammatory cell infiltration within the airways (Fig. 6c). A similar evolution was observed for the histological scoring of airways epithelium hypertrophy/hyperplasia, where values significantly higher than those observed in saline treated animals were still observable at 11 weeks (Fig. 5b), and similar results were observed upon quantitation of goblet cell scores (Fig. 5c). Representative histologies of Masson's trichrome stain and Alcian/PAS of lung sections obtained after 11 weeks of DRA are presented in Additional file 2: Figure S2.
Budesonide treatment inhibited by about $50 \%$ MMPSense680 fluorescent signal at 8 and 11 weeks (Fig. 6a, c), an effect that goes hand in hand with the observed inhibition of collagen deposition (Fig. 6b) and of the airways epithelium hypertrophy/hyperplasia (Fig. 5b).

\section{Discussion}

In the present study we provide evidence about the possibility to monitor in vivo in mice inflammatory cells infiltration and tissue remodeling associated with repeated 


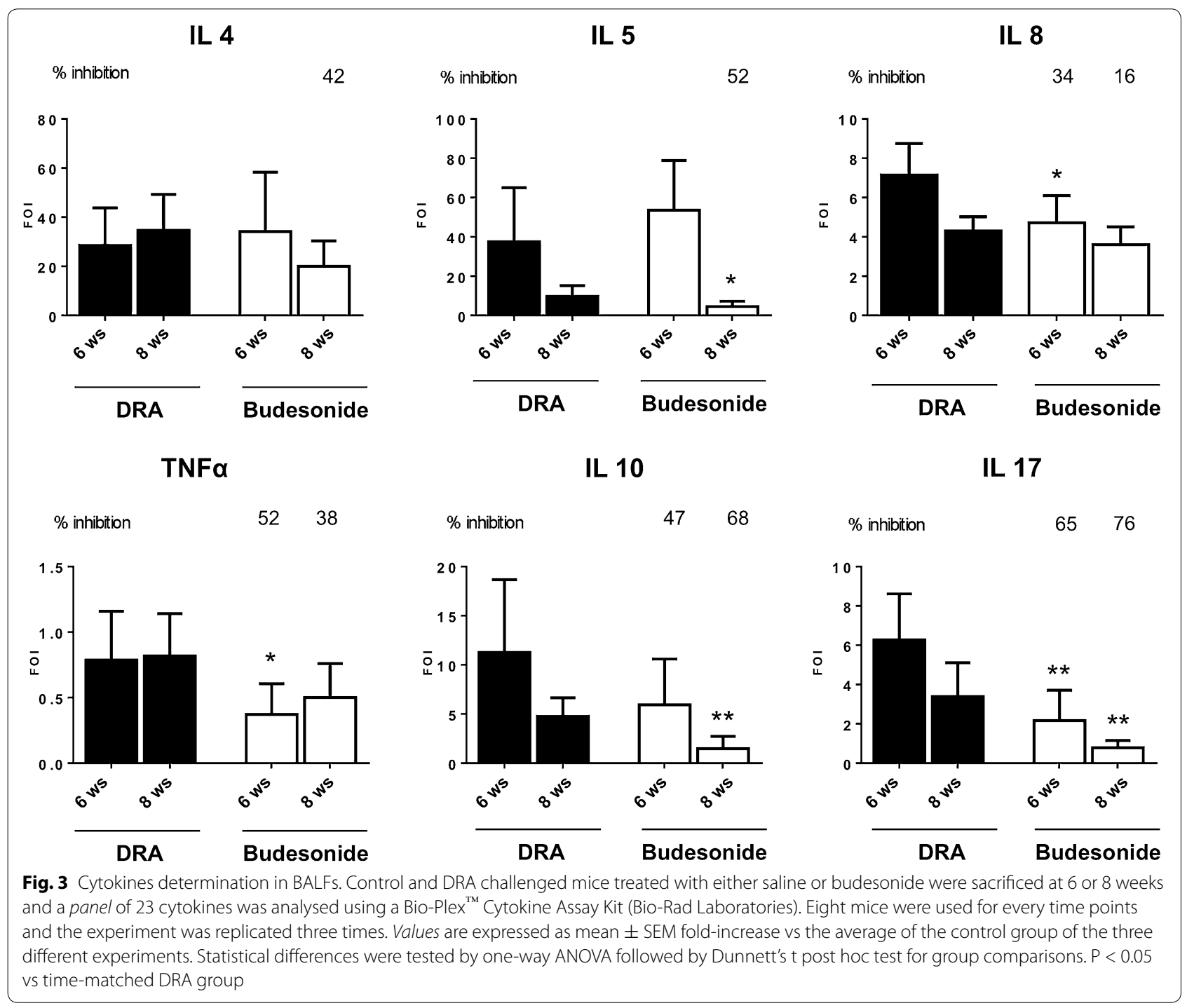

exposures to a combination of three natural allergens relevant for humans.

In agreement with previously published results [6] the present study confirms that multiple allergen exposure of mice breaks tolerance and induces a persisting airway pathology that is more closely mimicking the features of asthma in humans. Eight weeks of repeated allergen exposure cause a long lasting infiltration of inflammatory cells into the airways as well as pulmonary inflammation and histopathological lesions. Increased number of inflammatory cells such as eosinophils, neutrophils, monocytes/macrophages and lymphocytes were observed in BALFs and in pulmonary tissue up to 8 weeks after starting DRA administration, while collagen deposition, together with epithelial hyperplasia, were evident upon morphometric analysis at the later time points. Three weeks after stopping the exposure to allergen, cellular infiltration into the airways and the pulmonary tissue was almost completely resolved, but markers of the remodeling process such as collagen deposition, and airways epithelium hypertrophy/hyperplasia persisted, outlasting, as expected, the airways inflammatory cell infiltration [22].

Eosinophil infiltration is a hallmark of the pulmonary allergic inflammatory response, and markedly increased numbers of eosinophils were recovered in BALFs upon DRA administration. Eosinophil infiltration was very sensitive to the treatment with budesonide that strongly inhibited the increase in eosinophils both 2 and 4 weeks after the beginning of the treatment (that is after 6 and 8 weeks of DRA exposure). Interestingly, budesonide markedly decreased the concentrations of 


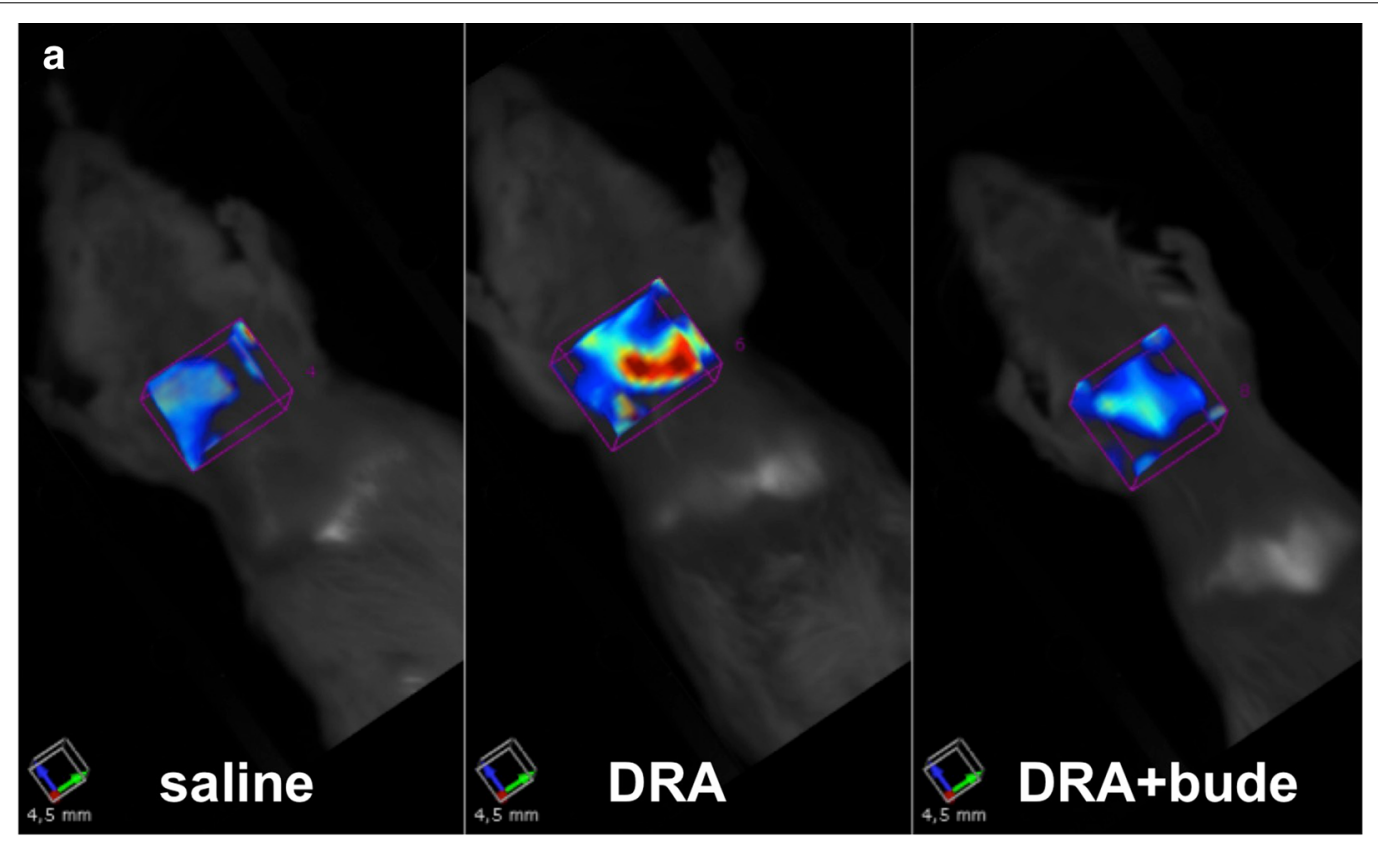

b

\section{ProSense 680}

- Saline

$\%$ inhibition

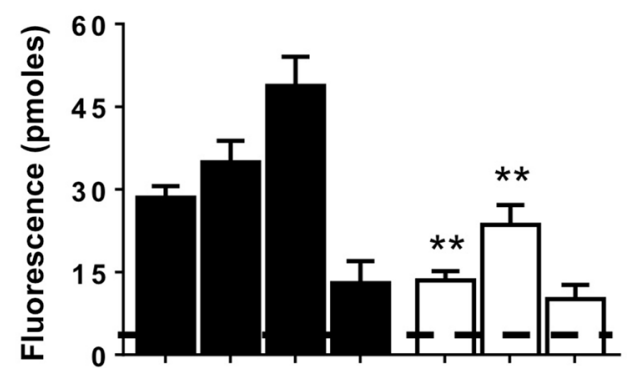

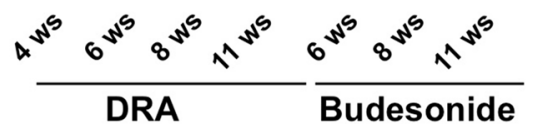

C

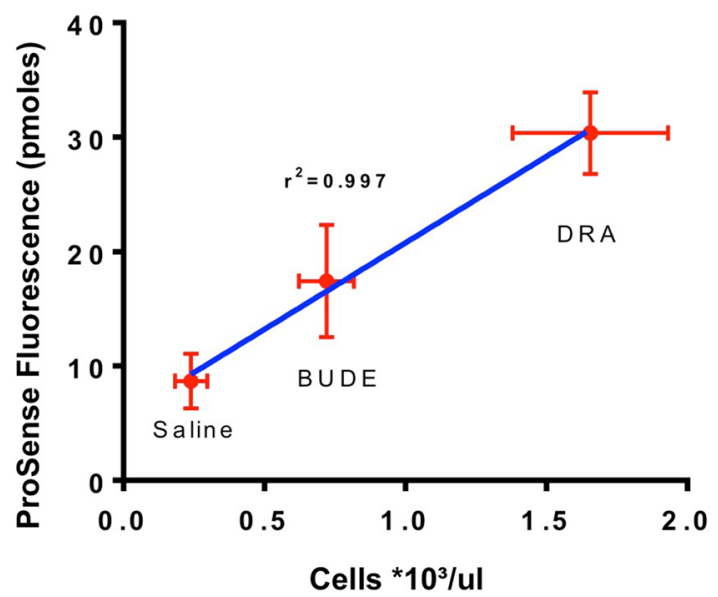

Fig. 4 a In vivo imaging of representative control, DRA-challenged and DRA-challenged and budesonide-treated mice injected with Prosense680 after 8 weeks of DRA. b Fluorescence signal quantification measured by FMT in ProSense680-injected mice at different time points after beginning of DRA, in the absence or presence of budesonide treatment beginning after 4 weeks of DRA. Values are expressed as mean \pm SEM of three different experiments. The dashed line represents the average value of control animals treated with saline. $\mathbf{c}$ Linear regression between the intensity of fluorescence resulting from ProSense680 and the total number of cell in BAL fluids from control, DRA-challenged and DRA-challenged and budesonide-treated mice injected with Prosense680 after 8 weeks of DRA. Eight mice were used for every time points and the experiment was replicated three times. Values are expressed as mean \pm SEM of the three different experiments. Statistical differences were tested by one-way ANOVA followed by Dunnett's t post hoc test for group comparisons. $P<0.05$ and ${ }^{* *} P<0.01$

the potent maturation, differentiation and activation factor for eosinophils IL-5 [23] only at the latest time point ( 8 weeks of DRA) but did not affect the increased concentrations of IL- 5 observed 2 weeks into the treatment with the topical corticosteroid (that is 6 week of DRA).
This result supports the notion that the effects of corticosteroid treatment are not simply mediated through the prevention of IL-5 release from cells within the lung, and is in agreement with a previous observation, obtained in a different mouse model of allergic asthma, showing the 

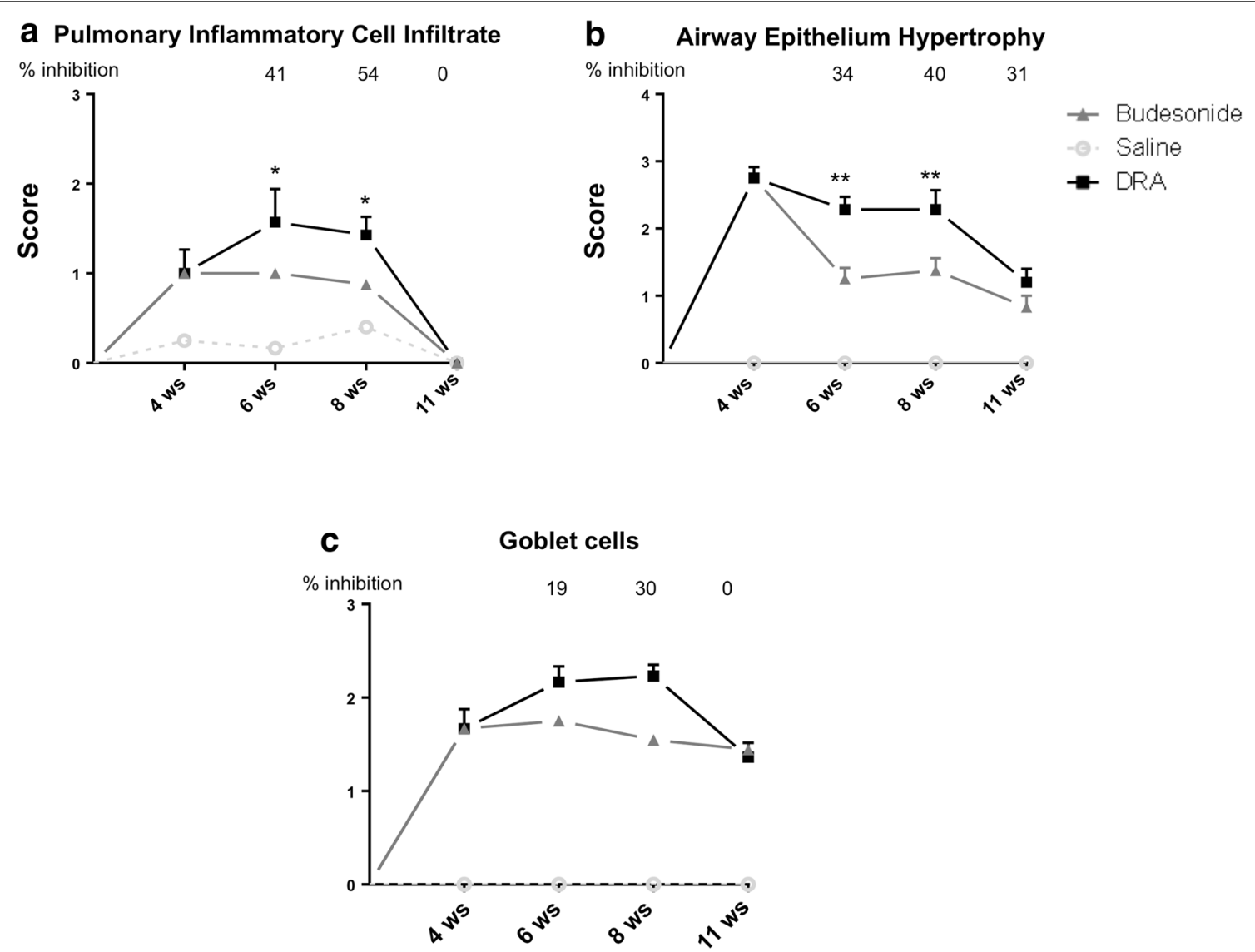

Fig. 5 a Pulmonary inflammatory cell infiltrate at different time-points after the beginning of DRA in the absence or presence of budesonide treatment beginning after 4 weeks of DRA. Eight mice were used for every time points and the experiment was replicated three times. Score values, determined as described in "Methods", are expressed as mean \pm SEM of the three different experiments. The dashed line represents the average value of control animals treated with saline. b Airway epithelium hypertrophy at different time-points after the beginning of DRA in the absence or presence of budesonide treatment beginning after 4 weeks of DRA. Eight mice were used for every time points and the experiment was replicated three times. Score values, determined as described in "Methods", are expressed as mean \pm SEM of the three different experiments. c Goblet cells quantitation at different time-points after the beginning of DRA in the absence or presence of budesonide treatment beginning after 4 weeks of DRA. Eight mice were used for every time points and the experiment was replicated three times. Score values, determined as described in "Methods", are expressed as mean \pm SEM of the three different experiments. The dashed line represents the average value of control animals treated with saline. Statistical analysis was carried out by one-way ANOVA followed by Fischer's exact test. $P<0.05$ and ${ }^{* *} P<0.01$

effect of corticosteroids on eosinophils preceding that on the concentrations of IL-5 [24].

Repeated exposure to low-dose allergen have been reported to increase the expression of CD11b on alveolar monocytes/macrophages, consistent with an influx of monocytes [25]; DRA exposure also resulted in an increased number of monocyte/macrophages in BALFs, together with an increased expression of the adhesion molecule CD11b, as a result of a raising proportion of monocytes in the alveolar macrophage population $[26,27]$.

Treatment with budesonide also reduced the number of neutrophils infiltrating into the airways, but this effect was only observed at the earliest observation point ( 2 weeks of budesonide treatment, 6 weeks of DRA), while no inhibition was evident after 4 weeks of treatment (8 weeks of DRA). This effect well correlated with the changes in BALFs concentrations of the potent neutrophil chemotactic factor IL-8 [28], and is in agreement with previously published results [29].

Recent breakthroughs in photonic technology have allowed the advance of optical imaging beyond standard, low penetration two-dimensional fluorescence reflectance imaging (FRI) to allow for three dimensional fluorescent tomographic in vivo imaging systems. Such technology has opened the door not only to deep-tissue detection and localization but also to the new capability of absolute quantification of fluorescence at any site within the body.

This and other optical imaging techniques could also be used in animal studies to validate probes, such as 


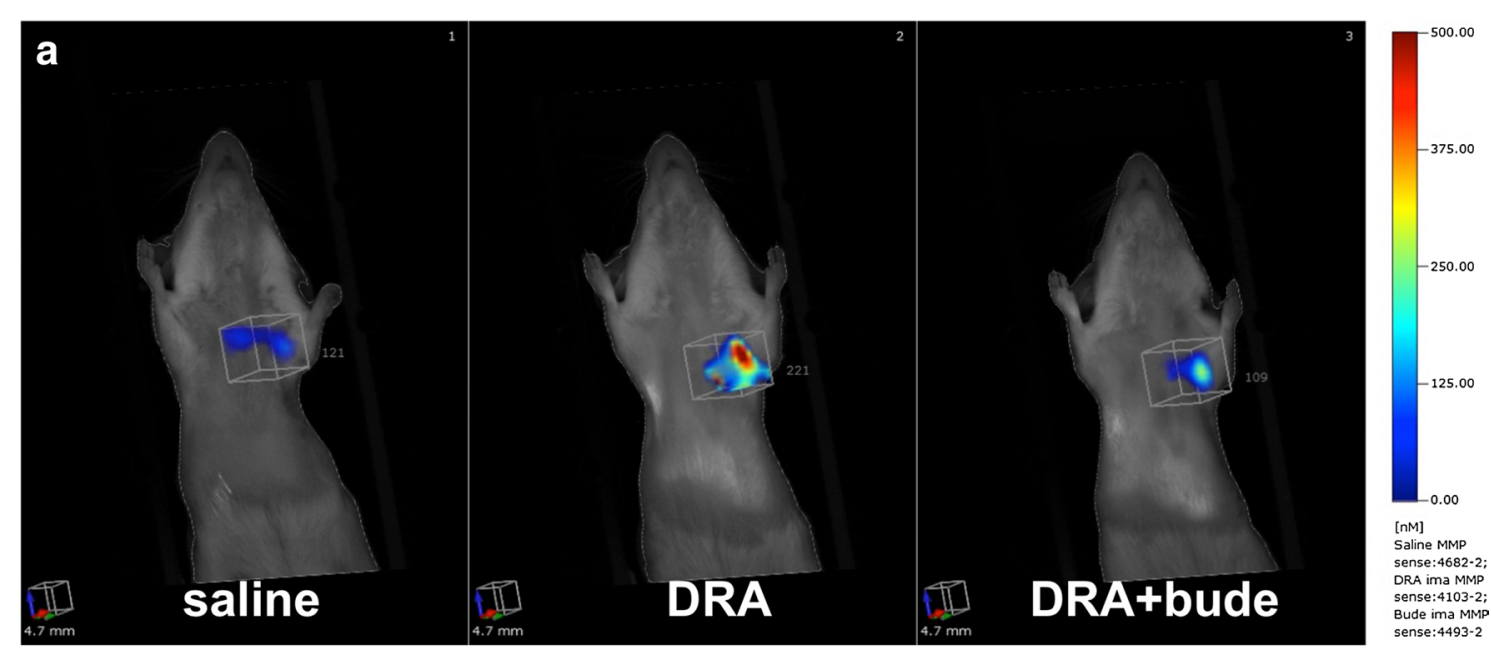

b Collagen deposition

- Saline

$\%$ inhibition

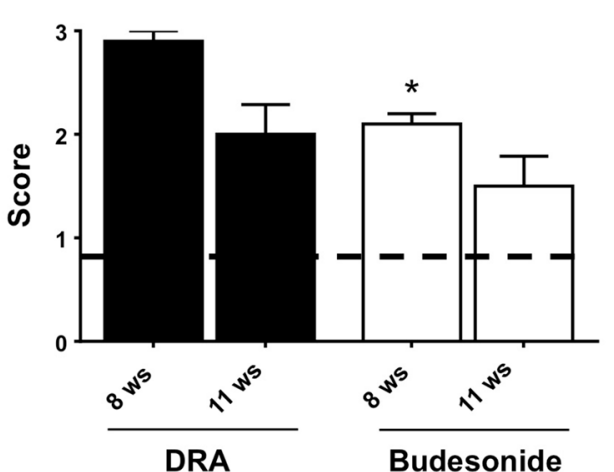

C

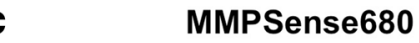

$\%$ inhibition

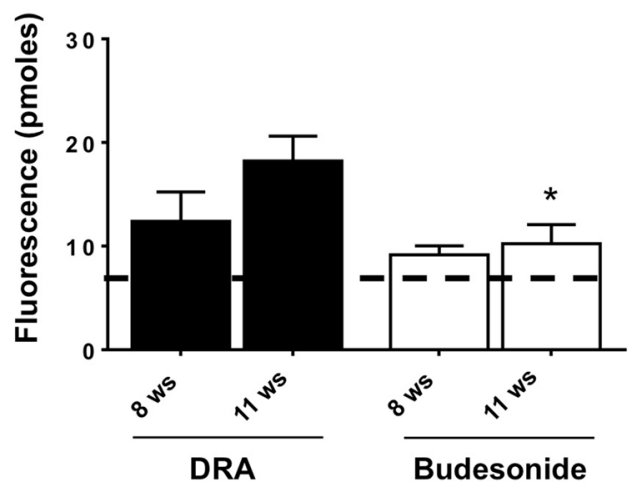

Fig. 6 a In vivo imaging of representative control, DRA-challenged and DRA-challenged and budesonide-treated mice injected with MMPSense680 after 11 weeks from the beginning of DRA administration (3 weeks after ending of DRA administration). $\mathbf{b}$ Score of collagen deposition measured by Masson's Trichrome staining at different time points after beginning of DRA, in the absence or presence of budesonide treatment beginning after 4 weeks of DRA. Values are expressed as mean \pm SEM of three different experiments. The dashed line represents the average value of control animals treated with saline. c Fluorescence signal quantification measured by FMT in MMPSense680-injected mice at different time points after beginning of DRA, in the absence or presence of budesonide treatment beginning after 4 weeks of DRA. Eight mice were used for every time points and the experiment was replicated three times. Values are expressed as mean \pm SEM of the three different experiments. The dashed line represents the average value of control animals treated with saline. Statistical differences were tested by one-way ANOVA followed by Dunnett's $t$ post hoc test for group comparisons. $P<0.05$ and ${ }^{* *} P<0.01$

peptides/antibodies that may then be used in humans by replacing the fluorescent marker with a radioisotope allowing nuclear medicine imaging [30].

Eosinophils are known to produce a variety of proteases and lysosomal cysteine proteases, including cathepsins B, $\mathrm{L}$, and $\mathrm{S}[31,32]$. Such proteases may be relevant in the pathogenesis of asthma and other pulmonary diseases, through their contribution to extracellular matrix degradation [33] and the modulation of immune responses [34]. Metalloproteinase (MMP) may also play a role in pulmonary diseases such as asthma, COPD and interstitial lung diseases, where an unbalance between their activity and the activity of endogenous inhibitors (tissue inhibitors of metalloproteinases: TIMPs) is considered a key event [35-37], and MMP inhibition is an attractive therapeutic target [38]. Structural cells such as fibroblasts, bronchial and alveolar epithelial cells, and smooth muscle cells [39-41] as well as inflammatory cells such as neutrophils, alveolar macrophages, mast cells and eosinophils [42-44] are known to synthesize MMP in the lung. 
Taking advantage of cathepsin-sensitive (ProSense680) and metalloproteinase-sensitive (MMPSense680) nearinfrared fluorescent agents associated with FMT, we monitored the activity of these proteinases within the lungs of mice at different times during the DRA administration, finding that fluorescent signal intensity well correlated with specific inflammatory responses, as assessed in parallel groups of animals. In particular, cathepsin activity provided results that quantitatively correlated with the number of inflammatory cells in BALFs, while MMP activity better correlated with remodeling processes such as collagen deposition. Although it cannot be ruled out that the correlations observed may only be circumstantial, the results obtained with post-mortem bronchoalveolar lavage (BAL) fluid analyses, cells counts, and histology at different time points all support the correlation between inflammatory cell infiltration/remodeling and the in vivo fluorescence signals observed,

Treatment with budesonide of mice undergoing DRA with already established pulmonary inflammation, decreased both the conventionally assessed inflammation parameters and cathepsin- and MMP-derived fluorescence, providing evidence that FMT allows the quantitative evaluation of the activity of corticosteroids in this recently described experimental model of asthma, both on inflammatory cells pulmonary infiltration and, at least partially, on tissue remodeling.

FMT using NIRF probes such as ProSense680 and MMSense 680 has been successfully used to assess pulmonary inflammation in LPS-treated $[45,46]$ and in ovalbumin-sensitized and challenged mice [46], as well as to evaluate the effect of corticosteroid in ovalbuminsensitized and challenged mice [46, 47]. More recently, alternative NIRF probes targeting selectin adhesion molecules have also been used to monitor the recruitment of inflammatory cells in ovalbumin-sensitized and challenged mice $[48,49]$ and, again, the efficacy of steroids in suppressing allergic inflammatory response in the lung [49].

Nevertheless in all these studies FMT was used to monitor acute inflammatory responses, either caused by sensitization and challenge or by exposure to LPS, and/or the effect of corticosteroid treatment on such a response. In our study, the use of a recently described chronic asthma model in mice together with in vivo FMT imaging, allowed real time evaluation of activities associated with chronic allergic inflammation in the lung, enabling longitudinal non-invasive consecutive examinations.

The long lasting allergic inflammatory response observed in this model also allowed the evaluation of the therapeutic intervention with topical budesonide, rather than the prophylactic activity of corticosteroid assessed in other studies [46, 47, 49]. Indeed, randomization to treated and control groups using the FMT results of ProSense680 carried out after 4 weeks of DRA administration, minimizes variability by equally representing, in the control and budesonide-treated group, animals with different responses to DRA, therefore maximizing the possibility to assess statistically significant differences associated to treatment using a limited number of animals.

In vivo FMT imaging allowed to monitor non invasively and throughout the 11 weeks of the protocol, different component of the pulmonary inflammation associated with this novel model of chronic asthma, overcoming one of the mayor limitations for the use of asthma mouse models where conventional assessments of inflammation and asthma progression rely on invasive measures of pulmonary function and ex vivo characterization of pulmonary cellular infiltration. Although these assessments of disease status are used extensively and highly validated, they are terminal procedures that preclude the possibility of repeated, longitudinal assessment of test subjects.

\section{Conclusions}

We studied a recently described, chronic asthma model resulting from an eight-week, twice a week, intranasal challenge with triple allergens (DRA) in female Balb/C mice. In addition to confirming that this model better reproduces the feature of human chronic asthma with inflammatory cell infiltrates associated with epithelial hyperplasia that last throughout the period of allergen administration and beyond, we also demonstrated the ability of the FMT imaging to non invasively monitor and quantify different components of the inflammatory response in the lung in a robust and consistent manner. The use of a cathepsin-sensitive agent produced results that well correlated with cellular infiltrates within the airways and the pulmonary tissue. On the other side the results obtained with a metalloproteinase-sensitive agent resulted in mirroring more closely collagen deposition and epithelial cell hypertrophy, therefore showing the potential to non-invasively monitor different component of the inflammatory response associated with DRA. The consistency of the quantitative tomography and its excellent correlation with BAL assessment and histological determinations provides a powerful tool for monitoring the disease and quantify the efficacy of therapeutic agents. FMT imaging applied to asthma research, using new and existing near infrared fluorescent imaging agents, may therefore provide a novel, non-invasive tool for understanding pulmonary inflammation and help develop new therapeutics. DRA model together with FMT will open up a new way to look at asthma progression and the pharmacological response to treatment of different classes of anti-inflammatory drugs. 


\section{Additional files}

Additional file 1: Figure S1. Ex vivo imaging of fluorescence from excised lungs obtained from mice receiving saline (Panel A) or DRA (Panel B) after administration of MMPSense680 at 11 weeks and from mice receiving saline (Panel C) or DRA (Panel D) after administration of ProSense 680 at 8 weeks.

Additional file 2: Figure S2 Representative images of histological slides obtained at 11 weeks from the lungs of mice receiving saline (Panel A) or DRA (Panel B) stained with Alcian/PAS, and from the lungs of mice receiving saline (Panel C) or DRA (Panel D) stained with Masson's Trichrome. Alcian/PAS and Masson's Trichrome staining were performed as described in "Methods".

\section{Abbreviations}

FMT: fluorescence molecular tomography; DRA: dust mite, ragweed, and aspergillus; BALFs: bronchoalveolar lavage fluids.

\section{Authors' contributions}

Conception and design, FS, AS, GV, CC, PM, MC Laboratory testing, FS, FR, SC. Data collection, FS, FR. Data analysis and interpretation: FS, FR, AS, GV Drafting of manuscript: FS, AS, GV, MV, MC. All authors read and approved the final manuscript.

\section{Author details}

${ }^{1}$ Chiesi Farmaceutici S.p.A, Parma, Italy. ${ }^{2}$ Dipartimento di Scienze Farmacologiche e Biomolecolari, Università degli Studi di Milano, Via Balzaretti 9, 20133 Milan, Italy. ${ }^{3}$ IBIM, Consiglio Nazionale delle Ricerche, Palermo, Italy. ${ }^{4}$ Dipartimento di Scienze Biomediche, Biotecnologiche e Traslazionali, Università di Parma, Parma, Italy.

\section{Acknowledgements}

This work was supported by Institutional funds from Chiesi Farmaceutici (FS, FR, CC, MC, GV) and from Università degli Studi di Milano (AS).

\section{Competing interests}

FS, CC, MC e GV are employees of Chiesi Farmaceutici S.p.A., that supported the research work. FR is a consultant of Chiesi Farmaceutici S.p.A.

Received: 22 June 2015 Accepted: 13 October 2015

Published online: 24 October 2015

\section{References}

1. Shin YS, Takeda K, Gelfand EW. Understanding asthma using animal models. Allergy Asthma Immunol Res. 2009;1:10-8.

2. Lommatzsch M, Julius P, Kuepper M, Garn H, Bratke K, Irmscher S, Luttmann W, Renz H, Braun A, Virchow JC. The course of allergen-induced leukocyte infiltration in human and experimental asthma. J Allergy Clin Immunol. 2006;118:91-7.

3. Whitehead GS, Walker JKL, Berman KG, Foster WM, Schwartz DA. Allergeninduced airway disease is mouse strain dependent. Am J Physiol Lung Cell Mol Physiol. 2003;285:L32-42.

4. Van Hove CL, Maes T, Joos GF, Tournoy KG. Prolonged inhaled allergen exposure can induce persistent tolerance. Am J Respir Cell Mol Biol. 2007;36:573-84.

5. Cui Z-H, Joetham A, Aydintug MK, Hahn Y-S, Born WK, Gelfand EW Reversal of allergic airway hyperreactivity after long-term allergen challenge depends on gammadelta T cells. Am J Respir Crit Care Med. 2003;168:1324-32.

6. Goplen N, Karim MZ, Liang Q, Gorska MM, Rozario S, Guo L, Alam R. Combined sensitization of mice to extracts of dust mite, ragweed, and Aspergillus species breaks through tolerance and establishes chronic features of asthma. J Allergy Clin Immunol. 2009;123(925-32):e11.
7. Bianchi A, Ozier A, Ousova O, Raffard G, Crémillieux Y. Ultrashort-TE MRI longitudinal study and characterization of a chronic model of asthma in mice: Inflammation and bronchial remodeling assessment. NMR Biomed. 2013:26:1451-9.

8. Lederlin M, Ozier A, Dournes G, Ousova O, Girodet PO, Begueret H, Marthan R, Montaudon M, Laurent F, Berger P: In Vivo Micro-CT Assessment of Airway Remodeling in a Flexible OVA-Sensitized Murine Model of Asthma. PLoS One 2012,7.

9. Blé F-X, Cannet C, Zurbruegg S, Karmouty-Quintana H, Bergmann R, Frossard N, Trifilieff A, Beckmann N. Allergen-induced lung inflammation in actively sensitized mice assessed with MR imaging. Radiology. 2008;248:834-43.

10. Blé FX, Cannet C, Zurbruegg S, Gérard C, Frossard N, Beckmann N, Trifilieff A. Activation of the lung S1P 1 receptor reduces allergen-induced plasma leakage in mice. Br J Pharmacol. 2009;158:1295-301.

11. Nakano Y, Muller NL, King GG, Niimi A, Kalloger SE, Mishima M, Pare PD. Quantitative assessment of airway remodeling using high-resolution CT. Chest. 2002;122(6 Suppl):271S-5S

12. Reinartz P, Kaiser H-J, Wildberger JE, Gordji C, Nowak B, Buell U. SPECT imaging in the diagnosis of pulmonary embolism: automated detection of match and mismatch defects by means of image-processing techniques. J Nucl Med. 2006;47:968-73.

13. Harris RS, Winkler T, Tgavalekos N, Musch G, Melo MFV, Schroeder T, Chang Y, Venegas JG. Regional pulmonary perfusion, inflation, and ventilation defects in bronchoconstricted patients with asthma. Am J Respir Crit Care Med. 2006;174:245-53.

14. Harris RS, Schuster DP: Visualizing lung function with positron emission tomography. J Appl Physiol (Bethesda, Md 1985) 2007, 102:448-458.

15. Enright $\mathrm{H}$ a, Bratt JM, Bluhm AP, Kenyon NJ, Louie AY. Tracking retention and transport of ultrafine polystyrene in an asthmatic mouse model using positron emission tomography. Exp Lung Res 2013; 39:304-13.

16. Thomas AC, Kaushik SS, Nouls J, Potts EN, Slipetz DM, Foster WM, Driehuys B. Effects of corticosteroid treatment on airway inflammation, mechanics, and hyperpolarized $3 \mathrm{He}$ magnetic resonance imaging in an allergic mouse model. J Appl Physiol 2012;1437-1444.

17. Crane LMA, Themelis G, Arts HJG, Buddingh KT, Brouwers AH, Ntziachristos $V$, van Dam GM, van der Zee AGJ. Intraoperative near-infrared fluorescence imaging for sentinel lymph node detection in vulvar cancer: first clinical results. Gynecol Oncol. 2011;120:291-5.

18. Van Dam GM, Themelis G, Crane LMA, Harlaar NJ, Pleijhuis RG, Kelder W, Sarantopoulos A, de Jong JS, Arts HJG, van der Zee AGJ, Bart J, Low PS, Ntziachristos $V$. Intraoperative tumor-specific fluorescence imaging in ovarian cancer by folate receptor-alpha targeting: first in-human results. Nat Med. 2011:17:1315-9.

19. Weissleder R, Ntziachristos $V$. Shedding light onto live molecular targets. Nat Med. 2003:9:123-8.

20. Institute of Laboratory Animal Research, Commission on Life Sciences, National Research Council. Guide for the care and use of laboratory animals. Washington, D.C.: National Academy Press; 1996. http://www. nap.edu/catalog/5140.html.

21. Stellari FF, Sala A, Donofrio G, Ruscitti F, Caruso P, Topini TM, Francis KP, Li X, Carnini C, Civelli M, Villetti G. Azithromycin inhibits nuclear factor-kB activation during lung inflammation: an in vivo imaging study. Pharmacol Res Perspect. 2014;2:e00058.

22. Al-Muhsen S, Johnson JR, Hamid Q. Remodeling in asthma. J Allergy Clin Immunol 2011;128:451-62 (quiz 463-4)

23. Weltman JK, Karim AS. IL-5: biology and potential therapeutic applications. Expert Opin Investig Drugs. 2000;9:491-6.

24. Shen H, O'Byrne PM, Ellis R, Wattie J, Tang C, Inman MD. The effects of intranasal budesonide on allergen-induced production of interleukin-5 and eotaxin, airways, blood, and bone marrow eosinophilia, and eosinophil progenitor expansion in sensitized mice. Am J Respir Crit Care Med. 2002;166:146-53.

25. Lensmar C, Prieto J, Dahlén B, Eklund A, Grunewald J, Roquet A. Airway inflammation and altered alveolar macrophage phenotype pattern after repeated low-dose allergen exposure of atopic asthmatic subjects. Clin Exp Allergy. 1999;29:1632-40.

26. Prieto J, Eklund A, Patarroyo M. Regulated expression of integrins and other adhesion molecules during differentiation of monocytes into macrophages. Cell Immunol. 1994;156:191-211. 
27. Stent G, Irving L, Lewin S, Crowe SM. The kinetics of surface expression of CD11/CD18 integrins and CD54 on monocytes and macrophages. Clin Exp Immunol. 1995;100:366-76.

28. Baggiolini M, Clark-Lewis I. Interleukin-8, a chemotactic and inflammatory cytokine. FEBS Lett. 1992;307:97-101.

29. Liu R, Bai J, Xu G, Xuan L, Zhang T, Meng A, Hou Q. Multi-allergen challenge stimulates steriod-resistant airway inflammation via NF-kBmediated IL-8 expression. Inflammation. 2013;36:845-54.

30. Sandanaraj BS, Kneuer R, Beckmann N. Optical and magnetic resonance imaging as complementary modalities in drug discovery. Future Med Chem. 2010:2:317-37.

31. Cimerman N, Mesko BP, Krasovec M, Suskovic S, Kos J. Serum concentration and circadian profiles of cathepsins $B, H$ and $L$, and their inhibitors, stefins A and B, in asthma. Clin Chim Acta. 2001;310:113-22.

32. Zheng T, Zhu Z, Wang Z, Homer RJ, Ma B, Riese RJ Jr, Chapman HA Jr, Shapiro SD, Elias JA. Inducible targeting of IL-13 to the adult lung causes matrix metalloproteinase- and cathepsin-dependent emphysema. J Clin Invest. 2000;106:1081-93.

33. Lalmanach G, Saidi A, Marchand-Adam S, Lecaille F, Kasabova M. Cysteine cathepsins and cystatins: from ancillary tasks to prominent status in lung diseases. Biol Chem. 2015;396:111-30.

34. Conus S, Simon HU. Cathepsins: key modulators of cell death and inflammatory responses. Biochem Pharmacol. 2008;76:1374-82.

35. Gueders MM, Foidart J-M, Noel A, Cataldo DD. Matrix metalloproteinases (MMPs) and tissue inhibitors of MMPs in the respiratory tract: potential implications in asthma and other lung diseases. Eur J Pharmacol. 2006;533:133-44.

36. Oikonomidi S, Kostikas K, Tsilioni I, Tanou K, Gourgoulianis KI, Kiropoulos TS. Matrix metalloproteinases in respiratory diseases: from pathogenesis to potential clinical implications. Curr Med Chem. 2009;16:1214-28.

37. Ohbayashi H. Matrix metalloproteinases in lung diseases. Curr Protein Pept Sci. 2002;3:409-21.

38. Vandenbroucke RE, Dejonckheere E, Libert C. A therapeutic role for matrix metalloproteinase inhibitors in lung diseases? Eur Respir J. 2011;38:1200-14.

39. Xu J, Benyon RC, Leir S-H, Zhang S, Holgate ST, Lackie PM. Matrix metalloproteinase-2 from bronchial epithelial cells induces the proliferation of subepithelial fibroblasts. Clin Exp Allergy. 2002;32:881-8.
40. Johnson S, Knox A. Autocrine production of matrix metalloproteinase-2 is required for human airway smooth muscle proliferation. Am J Physiol. 1999;277(6 Pt 1):L1109-17.

41. Elshaw SR, Henderson N, Knox AJ, Watson SA, Buttle DJ, Johnson SR. Matrix metalloproteinase expression and activity in human airway smooth muscle cells. Br J Pharmacol. 2004;142:1318-24.

42. Cataldo D, Munaut C, Noël A, Frankenne F, Bartsch P, Foidart JM, Louis R. Matrix metalloproteinases and TIMP-1 production by peripheral blood granulocytes from COPD patients and asthmatics. Allergy. 2001;56:145-51.

43. Ohno I, Ohtani H, Nitta Y, Suzuki J, Hoshi H, Honma M, Isoyama S, Tanno Y, Tamura G, Yamauchi K, Nagura H, Shirato K. Eosinophils as a source of matrix metalloproteinase-9 in asthmatic airway inflammation. Am J Respir Cell Mol Biol. 1997;16:212-9.

44. Baram D, Vaday GG, Salamon P, Drucker I, Hershkoviz R, Mekori YA. Human mast cells release metalloproteinase- 9 on contact with activated T cells: juxtacrine regulation by TNF-alpha. J Immunol. 2001;167:4008-16.

45. Haller J, Hyde D, Deliolanis N, de Kleine R, Niedre M, Ntziachristos V. Visualization of pulmonary inflammation using noninvasive fluorescence molecular imaging. J Appl Physiol. 2008;104:795-802.

46. Cortez-Retamozo V, Swirski FK, Waterman P, Yuan H, Figueiredo JL, Newton AP, Upadhyay R, Vinegoni C, Kohler R, Blois J, Smith A, Nahrendorf M, Josephson L, Weissleder R, Pittet MJ. Real-time assessment of inflammation and treatment response in a mouse model of allergic airway inflammation. J Clin Invest. 2008;1 18:4058-66.

47. Korideck H, Peterson JD. Noninvasive quantitative tomography of the therapeutic response to dexamethasone in ovalbumin-induced murine asthma. J Pharmacol Exp Ther. 2009;329:882-9.

48. Biffi S. Dal Monego S, Dullin C, Garrovo C, Bosnjak B, Licha K, Welker P, Epstein MM, Alves F: Dendritic polyglycerolsulfate near infrared fluorescent (NIRF) dye conjugate for non-invasively monitoring of inflammation in an allergic asthma mouse model. PLoS ONE. 2013;8:e57150.

49. Markus MA, Dullin C, Mitkovski M, Prieschl-Grassauer E, Epstein MM, Alves F. Non-invasive optical imaging of eosinophilia during the course of an experimental allergic airways disease model and in response to therapy. PLOS ONE. 2014;9:e90017.

\section{Submit your next manuscript to BioMed Central and take full advantage of:}

- Convenient online submission

- Thorough peer review

- No space constraints or color figure charges

- Immediate publication on acceptance

- Inclusion in PubMed, CAS, Scopus and Google Scholar

- Research which is freely available for redistribution

Submit your manuscript at

www.biomedcentral.com/submit
C Biomed Central 